\title{
Dynamic temperature estimation and real time emergency rating of transmission cables
}

Olsen, R. S.; Holboll, J.; Gudmundsdottir, Unnur Stella

Published in:

IEEE Power \& Energy Society General Meeting

Link to article, DOI:

10.1109/PESGM.2012.6345324

Publication date:

2012

Document Version

Publisher's PDF, also known as Version of record

Link back to DTU Orbit

Citation (APA):

Olsen, R. S., Holboll, J., \& Gudmundsdottir, U. S. (2012). Dynamic temperature estimation and real time emergency rating of transmission cables. In IEEE Power \& Energy Society General Meeting IEEE.

https://doi.org/10.1109/PESGM.2012.6345324

\section{General rights}

Copyright and moral rights for the publications made accessible in the public portal are retained by the authors and/or other copyright owners and it is a condition of accessing publications that users recognise and abide by the legal requirements associated with these rights.

- Users may download and print one copy of any publication from the public portal for the purpose of private study or research.

- You may not further distribute the material or use it for any profit-making activity or commercial gain

- You may freely distribute the URL identifying the publication in the public portal 


\title{
Dynamic Temperature Estimation and Real Time Emergency Rating of Transmission Cables
}

\author{
R. S. Olsen, J. Holboll and U. S. Gudmundsdóttir
}

\begin{abstract}
This paper is concerned with the development of a fast computational methodology for dynamical estimation of the temperature in transmission cables solely based on current measurements and an enhanced version of the lumped parameters model, also denoted thermo electric equivalents (TEE). It is found that the calculated temperature estimations are fairly accurate - within $1.5^{\circ} \mathrm{C}$ of the finite element method (FEM) simulation to which it is compared - both when looking at the temperature profile (time dependent) and the temperature distribution (geometric dependent). The methodology moreover enables real time emergency ratings, such that the transmission system operator can make well-founded decisions during faults. Hereunder is included the capability of producing high resolution loadability vs. time schedules within few minutes, such that the TSO can safely control the system.
\end{abstract}

Index Terms--Cables, transmission lines, dynamic temperature control, finite element methods, temperature prediction methods.

\section{INTRODUCTION}

$\mathrm{T}$ HE present paper will introduce a methodology for fast estimation of dynamic temperatures in transmission cables, as well as enabling online dynamic temperature predictions.

The generally accepted method for rating of transmission cables follows the IEC 60287 standard [1], which is used to calculate the ampacity/loadability under steady state and worst case conditions. The limiting parameter for loadability of cables is the temperature, either at the conductor or at the jacket; however transmission systems are operated on the basis of power and voltage, and the loadability of a cable is therefore not given as a temperature but as a current.

It is implied by the term steady state rating that the thermal inertia is neglected in the equations and the temperature will thus, most of the time, be well below the steady state rating. Furthermore, the steady state current rating includes, as stated, the worst case conditions. These worst case conditions will only rarely, if ever, be fulfilled, and the cable will therefore for the largest part of its lifetime be underutilised. These facts are well acknowledged and researchers have therefore tried to develop dynamic thermal rating (DTR) methods for cable

R. S. Olsen is with Section of Transmission Lines, Energinet.dk, Fredericia 7000, Denmark (e-mail: rso@energinet.dk).

J. Holboell is with the Department of Electrical Engineering, Technical University of Denmark, Kgs. Lyngby 2800, Denmark (e-mail: jh@elektro.dtu.dk).

U. S. Guðmundsdóttir is with Section of Transmission Lines, Energinet.dk, Fredericia 7000, Denmark (e-mail: usg@energinet.dk). systems. Some of these methods utilise finite element methods (FEM) (e.g. [2]), some are concerned with online temperature measurements (e.g. [3]) and yet again some utilise analytical methods (e.g. [4]).

The standards focus mainly on steady state calculations; however some dynamics are included [5], as they are concerned with both cyclic and emergency ratings. Again, these ratings are given as currents.

For doing these rating calculations, the standards utilise the resemblance between current flowing in an electric circuit, and heat flowing in a thermal circuit, the so called thermo electric equivalents (TEEs), based on electrical lumped parameter models. In [5] the TEEs are developed to consist of two loops, one for the internal part of the cable and one for the cable surroundings. This simplification allows for distinguishing between slow and fast temperature changes.

It is the intention of the present study to develop a general methodology which can be used for estimating the temperature in transmission cables dynamically, without distinguishing between slow and fast temperature changes.

Moreover, the study should develop tools for calculation of real time emergency ratings, which can help the operator of the transmission system to make fast choices during faults.

It is furthermore the aim that the model should be independent of temperature measurements, the computational time should be low and the method should be able to predict the temperature in the transmission cable solely based on estimated loading profiles.

Unless otherwise stated, the authors have used Matlab for calculations and production of graphical elements.

All Matlab simulations have been run on a $3.1 \mathrm{GHz}$ four processor, 64-bit Windows server 2008 with 4GB of ram.

All finite element simulations have been run on a $2.7 \mathrm{GHz}$ quad-core, 64-bit Windows $7 \mathrm{PC}$ with $8 \mathrm{~GB}$ of ram.

\section{Methodology}

As mentioned, there are a number of different approaches to take when the goal is to estimate temperatures in cable systems.

Firstly, FEM can in principle give results almost as accurate as one wishes, the size of the elements in the simulation can simply be made 'infinitely' small and all subcomponents can be included to a very detailed level. However the computational time is highly dependent on the number of 
elements in the model, and the simulation can therefore become very long, even for relatively simple systems.

The second option is to use the semi-analytical approaches as done in [4]. This approach is capable of calculating the continuous thermal field in the insulation by assuming simple boundary conditions. According to [4], the method is fast but some inaccuracies must be accepted. There is a risk that the simplifications, which are necessarily made with respect to boundary conditions, will result in accumulated errors, which will especially arise when a continuously varying load is imposed on the cable and not just a single current step.

A number of other methods have been investigated, such as e.g. the Finite Difference Method (FDM), time series analysis, etc. However the only serious competitor to the previously two described methods, as it is seen by the authors, is to enhance the models proposed by IEC in e.g. [5]. The computational time is expected to be low compared to FEM, but the drawback is a possible less accurate calculation. One can argue that TEEs are simplified equivalents of FEM models; however FEM models are capable of easily implementing unsymmetrical parts, which is difficult in TEEs. The implementation of multiple cables is necessarily very simplified in the TEEs, whereas the FEM approach enables highly accurate implementation.

The lumped parameters model has proven sufficiently accurate for steady state analysis and to some degree it is possible to extend this accuracy to dynamic models also. As compared to the time demanding FEM simulations, the expected low computational time, required by TEE, introduces the perspective of including and utilising thermal calculations into entirely new applications in transmission system operation. In the following, FEM will only be used for parameter estimation and model verification.

TEEs are used e.g. in [1] for determination of the steady state ampacity and in e.g. [5] for cyclic and emergency ratings. However, the present study is not concerned with cyclic or emergency ratings, but true dynamic temperature estimations. It is therefore not sufficient to use the two loop TEEs which are suggested in [5].

As described, the concept of TEE is built on the resemblance between heat flowing in a thermal circuit and current flowing in an electric circuit. This means that thermal capacitances will be implemented as electrical capacitances in the TEE, and the thermal resistances will be inserted as electrical resistances. The heat sources in the different subcomponents of the cable will be implemented into the TEE as current sources. In the present study heat sources have been implemented at the conductor $\left(W_{c}\right)$, in the dielectric (the dielectric losses are split into two: $W_{d 1}$ and $W_{d 2}$ ) and in the screen $\left(W_{s}\right)$.

Creating the dynamic TEE in this way makes it possible to follow the temperatures at the different subcomponent interfaces. The temperatures will appear in the TEE as voltages at the different nodes.

In Fig. 1 the simplest of such a TEE is shown for an unarmoured single phase cable.

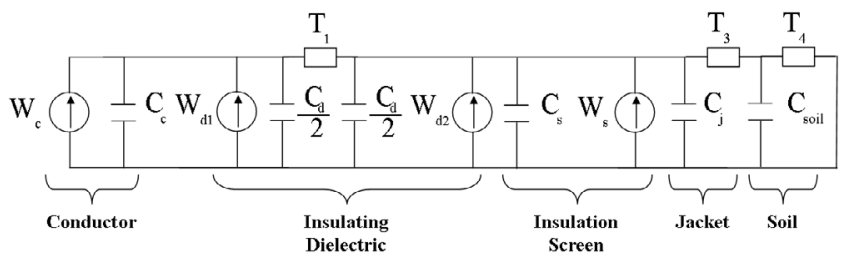

Fig. 1: The simplest thermo electric equivalent (TEE) of an unarmoured cable.

As it is seen from the figure, the thermal resistances of the metallic parts of the cable are neglected. This is assumed valid as the thermal resistivity of metals is usually two orders of magnitude smaller than the thermal resistivity of other parts of the cable.

It can be noticed from the above description and Fig. 1, that smaller subcomponents, such as semi conducting layers, are not included in the model. This is not due to restrictions in the methodology, but merely for easing the explanation of the proposed procedure.

The circuit in Fig. 1 is suitable for steady state and dynamic temperature estimation under constant and varying currents leaving the sources.

The proposed methodology has the inherent property of increasing the accuracy of the model with the cost of increased calculation time. By dividing the different subcomponents into several $\pi$-sections, a more accurate estimation of the temperature profile becomes available. This is an important feature, as the user of the model can choose the accuracy which is sufficient, and still have the benefit of high computational speed.

In clause III. it is shown why it can be important to utilise the possibility of increasing the accuracy of the model.

Considering simulation of the behaviour of a TEE circuit, it was chosen to solve the differential equations of the TEE at all nodes analytically [6], which means that the solution time can become very low.

The differential equations defining the 3-node system of Fig. 1 have for clarity been reduced to the matrix form as seen in (1).

An analytical solution requires the calculation of the Eigen values and Eigen vectors for the system matrix of (1).

$$
\left[\begin{array}{l}
\dot{\theta}_{e} \\
\dot{\theta}_{s} \\
\dot{\theta}_{c}
\end{array}\right]=\left[\begin{array}{ccc}
-\left(\frac{1}{C_{4} T_{3}}+\frac{1}{C_{4} T_{4}}\right) & \frac{1}{C_{4} T_{3}} & 0 \\
\frac{1}{C_{3} T_{3}} & -\left(\frac{1}{C_{3} T_{1}}+\frac{1}{C_{3} T_{3}}\right) & \frac{1}{C_{3} T_{1}} \\
0 & \frac{1}{C_{1} T_{1}} & -\frac{1}{C_{1} T_{1}}
\end{array}\right] \cdot\left[\begin{array}{l}
\theta_{e} \\
\theta_{s} \\
\theta_{c}
\end{array}\right]+\left[\begin{array}{l}
\frac{1}{C_{4} T_{4}} \cdot \theta_{a m b} \\
\frac{1}{C_{3}} \cdot\left(W_{d 2}+W_{s}\right) \\
\frac{1}{C_{1}} \cdot\left(W_{c}+W_{d 1}\right)
\end{array}\right]
$$

Here $\theta_{a m b}, \theta_{e}, \theta_{s}$ and $\theta_{c}$ are the ambient (above ground), jacket, screen and conductor temperatures respectively, and we define three capacitances:

$$
\begin{aligned}
& C_{I}=C_{c}+C_{d} / 2 \\
& C_{3}=C_{d} / 2+C_{s}+C_{j} \\
& C_{4}=C_{\text {soil }}
\end{aligned}
$$

The indices of these capacitances are: Conductor (c), dielectric $(d)$, screen $(s)$, jacket $(j)$ and surrounding soil (soil).

The thermal resistances $(T)$ are defined according to their physical position in the cable as it is seen in Fig. 1 and given in [7]. 
The solution to (1) is found by calculating the proper Eigen values $\left(\lambda_{1}, \lambda_{2}\right.$ and $\left.\lambda_{3}\right)$ and Eigen vectors $\left(v_{1}, v_{2}\right.$ and $\left.v_{3}\right)$. According to e.g. [6], the solution to such an equation system is as given in (2).

$$
\left[\begin{array}{l}
\theta_{e}(t) \\
\theta_{s}(t) \\
\theta_{c}(t)
\end{array}\right]=c_{1} \bar{v}_{1} \cdot e^{\lambda_{1} t}+c_{2} \bar{v}_{2} \cdot e^{\lambda_{2} t}+c_{3} \bar{v}_{3} \cdot e^{\lambda_{3} t}+\left[\begin{array}{l}
\theta_{e}(\infty) \\
\theta_{s}(\infty) \\
\theta_{c}(\infty)
\end{array}\right]
$$

The steady state temperatures at infinite time can be easily calculated by using the circuit of Fig. 1 and discarding the thermal capacitances.

The three constants $\left(c_{1}, c_{2}\right.$ and $\left.c_{3}\right)$ are determined by using the initial temperatures, which can be assumed to be equal to the ambient temperature if no previous load has been applied to the cable.

It should be noted that by increasing the accuracy of the model by splitting the subcomponents into more $\pi$-sections, the number of nodes will increase, thus the size of the system matrix of (1) and the computational time will also increase.

\section{A. Parameter Sizes}

In the present study, the thermal resistances $\left(T_{i}\right)$ are calculated according to the suggestions of [7]. The thermal resistivities $\left(\rho_{i, \text { thermal }}\right)$ of the individual layers are in this reference multiplied by a factor $\left(G_{\mathrm{i}}\right)$ depending on the geometry of the cable. The thermal resistance is therefore calculated as it is rephrased in (3), where $i$ is the layer (e.g. dielectric, jacket, etc).

$$
T_{i}=\frac{\rho_{i, \text { thermal }}}{2 \pi} \cdot G_{i}
$$
by:

The losses in the conductor and dielectric are simply given

$$
\begin{aligned}
& W_{c}=R_{\theta, A C} \cdot I^{2} \\
& W_{d}=2 \cdot \pi \cdot f \cdot C_{e l} \cdot U_{0}^{2} \cdot \tan (\delta)
\end{aligned}
$$

$R_{\theta, A C}$ being the temperature dependant AC resistance, $I$ is the current, $f$ is the system frequency, $C_{e l}$ is the electrical capacitance of the cable, $U_{0}$ is the phase to ground voltage and $\tan (\delta)$ is the dielectric loss factor.

It is in [1] assumed that the screen losses can be assessed directly as a fraction of the conductor losses. This approach is therefore also used in the present study. However the formulae used for the calculation of this quantity is highly dependent on the screen type, laying condition and bonding configuration. It is also recognised in [1] that the losses, under certain conditions, can become very small or even negligible.

The thermal capacitances are calculated by using the geometric parameters of the cable, given by the manufacturers, and utilising specific heat capacities typical for the materials involved.

The thermal capacitance and thermal resistance of the surrounding soil are usually the difficult quantities to determine due to their size and unsymmetrical heat flow.

Models are available for the thermal resistance of cable surroundings, but the authors have found only limited literature within the area of thermal capacitance. Furthermore, the models for thermal resistance are mainly aiming at steady state conditions. For that reason models on how to divide the soil equivalent into more $\pi$-sections is not thoroughly described in the literature.

On that background, it was chosen here to model the thermal capacitance of the soil with a cylinder centred at the centre cable and with a radius equal to the laying depth. The assumption is that such a cylinder will overestimate the thermal capacitance, compared to what will be experienced in real life. The heat will, in real life, seek mainly to the surface of the ground, whereas in the thermo electric equivalent, the heat is approximated to be distributed symmetrically away from the cable. This difference will result in the cylindrical approximation of the thermal capacitance of the soil being an overestimation, when comparing to what will be seen in real life experiments. The quantification of this overestimation and more suitable soil equivalents are left for future research.

The thermal resistance of the soil is known as varying a lot and being dependent on a number of parameters, e.g. moisture content. Also some laying configurations promote better thermal conditions than other. However the publications describing the thermal resistance of the soil are, as stated, almost solely concerned with steady state conditions (see e.g. [7] and [8] for extensive descriptions). On the basis of these steady state quantities, the present study uses some approximations in order to calculate the thermal conditions. For three single phase cables with an outer diameter of $D_{e}$, laid in flat formation with axial spacing of $s_{l}$, installed in a depth of $L$ and in soil with a thermal resistivity of $\rho_{\text {soil,therm }}$, the thermal resistance $T_{4}$ can, according to [7], be calculated as follows.

$$
T_{4}=\frac{\rho_{\text {soil }, \text { therm }}}{2 \cdot \pi} \cdot\left(\ln \left(u+\sqrt{u^{2}-1}\right)+\ln \left(1+\left(\frac{2 \cdot L}{s_{1}}\right)^{2}\right)\right)
$$

Where:

$u=2 L / D_{e}$

Equation (6) reduces to (7) when $u$ is much larger than unity, which is a normal condition.

$$
T_{4}=\frac{\rho_{\text {soil } \text { therm }}}{2 \cdot \pi} \cdot\left(\ln (2 \cdot u)+\ln \left(1+\left(\frac{2 \cdot L}{s_{1}}\right)^{2}\right)\right)
$$

\begin{tabular}{|c|c|c|c|c|c|}
\hline Material: & $\begin{array}{c}\tan \delta \\
{[]}\end{array}$ & $\begin{array}{c}\rho_{\text {electrical }} \\
{[\mu \Omega \cdot \mathrm{m}]}\end{array}$ & $\begin{array}{c}\alpha_{\text {electrical }} \\
{\left[\frac{\Omega}{\mathrm{K}}\right]}\end{array}$ & $\begin{array}{l}\mathrm{c}_{\text {thermal }} \\
{\left[\frac{\mathrm{J}}{\mathrm{m}^{3} \cdot \mathrm{K}}\right]}\end{array}$ & $\begin{array}{l}\rho_{\text {thermal }} \\
{\left[\frac{\mathrm{K} \cdot \mathrm{m}}{\mathrm{W}}\right]}\end{array}$ \\
\hline XLPE & 0.005 & - & - & $2.40 \cdot 10^{6}$ & 3.5 \\
\hline Aluminium & - & $2.83 \cdot 10^{-2}$ & $4.03 \cdot 10^{3}$ & $2.43 \cdot 10^{6}$ & 0 \\
\hline Copper & - & $1.72 \cdot 10^{-2}$ & $3.93 \cdot 10^{3}$ & $3.35 \cdot 10^{6}$ & 0 \\
\hline Soil & - & - & - & $1.92 \cdot 10^{6}$ & 1 \\
\hline
\end{tabular}

In the present study, the material specific parameters of Table 1 have been used.

TABLE 1

MATERIAL SPECIFIC PARAMETERS USED IN THE PRESENT STUDY

\section{RESUlts}

Results achieved by means of the analytical TEE approach were verified by comparison with a finite element model created in the commercial software Comsol Multiphysics version 4.2. Later, dynamic temperature measurements performed on real cables will be used for further verification.

The cable type modelled in the present paper is: single phase, $1000 \mathrm{~mm}^{2}$ compacted aluminium conductor, XLPE 
$420 \mathrm{kV}$ insulation, copper wire screen with a total cross sectional area of $200 \mathrm{~mm}^{2}$ and aluminium water blocking foil. The geometric quantities of the cable have been obtained from the NKT product catalogue [9]. The manufacturer's reference for this cable type is '1-leder PEX-AL-LRT $420 \mathrm{kV}$ '. The important cable geometries are given in Table 2.

$$
\text { TABLE } 2
$$

GEOMETRIC PARAMETERS OF CABLE UNDER INVESTIGATION.

\begin{tabular}{|c|c|c|c|c|}
\hline Component & Conductor & Insulation & Screen & Jacket \\
\hline $\begin{array}{c}\text { Outer diameter } \\
{[\mathrm{mm}]}\end{array}$ & 34 & 109.6 & 120.4 & 131.6 \\
\hline
\end{tabular}

The cables are laid in flat formation with $70 \mathrm{~mm}$ of spacing in between neighbouring cables and laid in a depth of 700 $\mathrm{mm}$.

\section{A. Models}

\section{1) Finite Element Model}

The FEM model is designed in 2D as seen in Fig. 2.

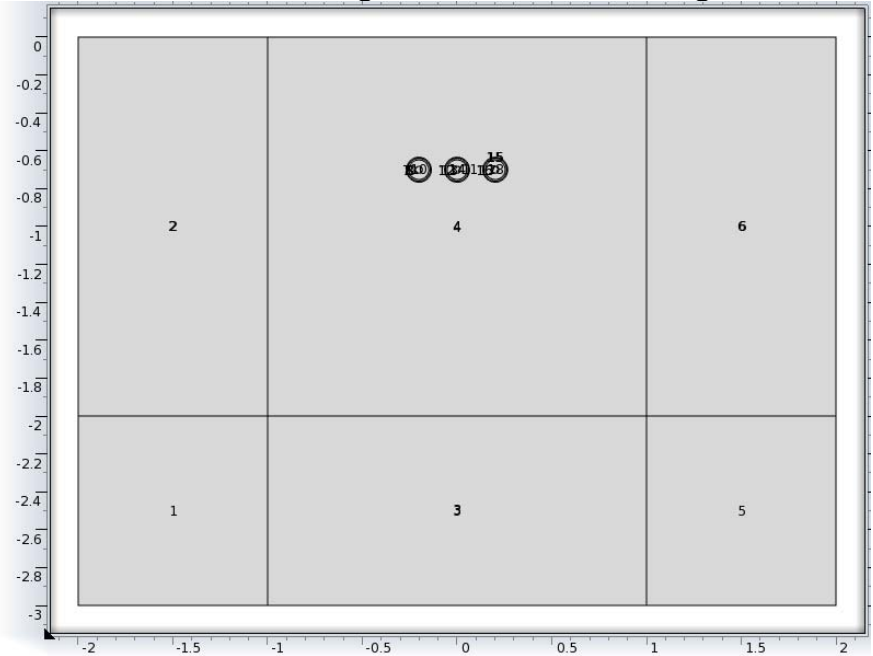

Fig. 2: Finite element model as designed in Comsol Multiphysics 4.2. The axes show the size in meters. The soil is an assembly of a normal rectangular part and infinite elements. The infinite elements limit the effects of the boundary conditions very much. The surface of the soil is assumed to be at $15^{\circ} \mathrm{C}$ at all sides.

The cables are modelled as four subcomponents, a solid aluminium conductor, XLPE insulation, solid copper screen and XLPE jacket. Conductor and screen are modelled as solids, which is done for simplicity and it is assumed that only limited errors will be introduced when only thermal equations are to be solved. Comsol is capable of combining the electric and thermal physics, however in the present study, focus is on validating the thermal models, and therefore only the thermal physics in Comsol are utilised. The size of the heat inputs are therefore calculated based on the equations given in [1].

The soil is in Comsol modelled as a large square part (denoted 4 on the figure) in combination with the infinite elements $(1,2,3,5$ and 6$)$, which limit the boundary effects.

It may appear that the model is small, so in order to confirm the sufficiency of it, a larger version was modelled as well, which gave identical results.

\section{2) Thermo Electric Equivalents}

The thermo electric equivalents of this study are designed according to the description of the preceding sections.

It is known that the thermal properties of the insulating material have a large impact during fast load changes (see e.g. the discussion on Van Wormer coefficients in [8]), why a division of the dielectric into multiple $\pi$-sections is reasonable. However for longer lasting load changes, the surrounding material will also have a large impact, why it is equally reasonable to divide the soil into multiple $\pi$-sections.

As this study is primarily concerned with dynamic load conditions the authors have focused on the long time scale. The remaining of this paper therefore deals with $\pi$-sectioning of the surrounding material, but it is to be noted that in order for the model to become more accurate, especially for fast load changes, $\pi$-sectioning of the dielectric may be highly necessary.

Regarding the division in $\pi$-sections, the laying depth was divided into $k$ equally large intervals as shown in Fig. 3. In Fig. $3 k$ is equal to three. It is seen that the thermal capacitances in this case must be calculated via the specific heat and the volume of hollow cylinders. In the figure is, for simplicity, shown the single cable case.

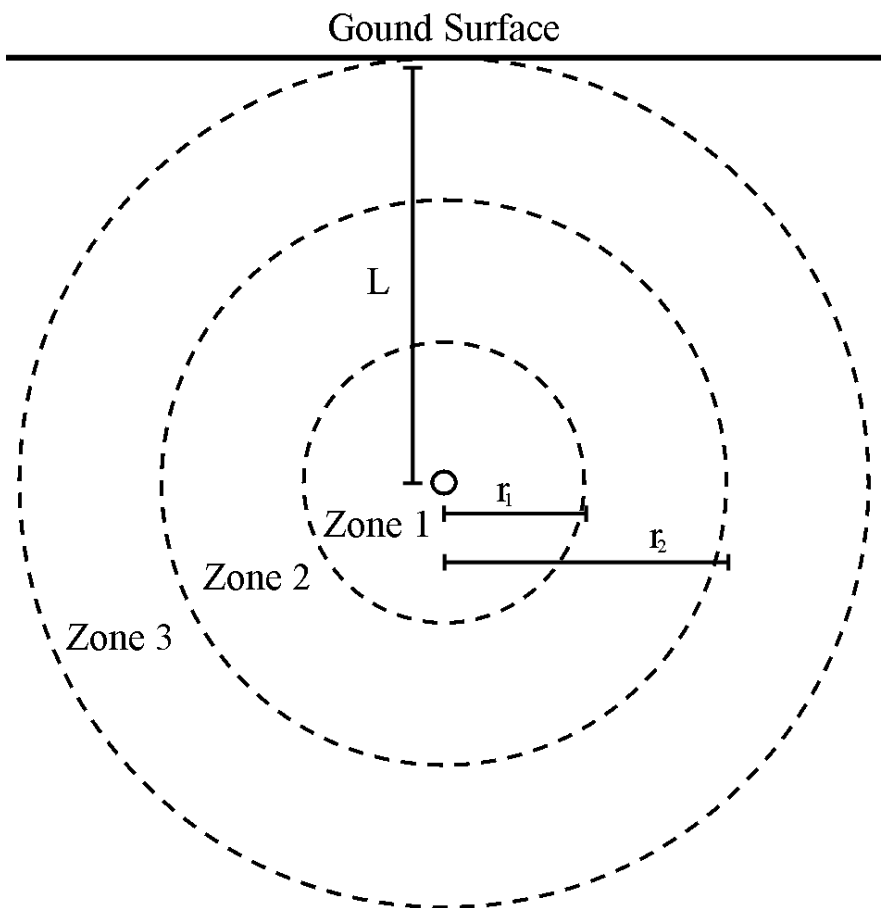

Fig. 3: The thermal resistance of the cable surroundings is divided into zones. In this figure a single cable is shown for simplicity. The soil is seen to be divided into three resistance parts.

The thermal resistance of the individual $\pi$-sections has been calculated based on adapted versions of the steady state equations of e.g. [8]. It should be noticed that the thermal resistance of Zone 3 is not equal to that of Zone 1 because the ratio of the radii is smaller. It is thus not sufficient simply to divide the thermal resistance $T_{4}$, as calculated by (7), with the number of intervals, $k$. 
Equation (7) is therefore used in an adapted version to calculate the thermal resistance of the individual zones. The thermal resistance of Zone ' $x$ ' (for the simple model of Fig. 3, $x$ can be the numbers 1,2 or 3 ) has in the present study been calculated as seen in (8).

$$
T_{4, x}=\frac{\rho_{\text {soil, therm }}}{2 \cdot \pi} \cdot\left(\ln \left(\frac{r_{x}}{r_{x-1}}\right)+\frac{\ln (2)+\ln \left(1+\left(\frac{2 \cdot L}{s_{1}}\right)^{2}\right)}{k}\right)
$$

It is seen from (8) that the thermal resistance is broken into two parts. The first part, related to the first logarithmic term, is concerned with the thermal resistance caused by the size of the zone. The second part, related to the second and third logarithmic terms, is concerned with the influence of the neighbouring cables.

It is seen from (8) that the first part of the formulae takes care of the difference in thermal resistance for the different zones. On the other hand, the second part of (8) shows that the present study assigns an equal quantity of the influence of the neighbouring cables for all zones. The latter is an approximation, however for simplicity, this approach has been chosen.

It should moreover be noted that the thermal parameters are assumed constant throughout the modelled timeframe. This means e.g. that no dry out of the soil is assumed. Again it is emphasized that this is not a limit enforced by the methodology, as $\rho_{\text {soil,therm }}$ can easily be made dependent on the distance from the cable $\left(r_{x}\right)$, but that the assumption is included for simplifying the explanation of the model.

In the following clauses, the sensitivity of the above described $\pi$-section division is tested.

The internal parts of the cable are, for simplicity, modelled as seen in Fig. 1. This means that no additional $\pi$-section division is performed for the cable subcomponents. It was considered to include Van Wormer coefficients as they are given in e.g. [8], however these coefficients change according to whether the transient is short or long lasting, and an inclusion in a dynamic study, such as the present, is therefore not meaningful.

To increase accuracy of the modelled thermal behaviour, the authors suggest that the thermal parameters, of the internal parts of the cable, can be divided into more $\pi$-sections in a similar way as it is done for the soil in (8). In this way it will also be possible to distribute the dielectric losses more evenly throughout the insulation. However, as mentioned, for simplicity, $\pi$-sectioning is used on the surrounding soil only.

\section{B. Load Profile}

The cables have in the present study been subjected to the load profile seen in Fig. 4. It has been chosen to vary the load in steps; however this is not a limitation in the methodology of TEE.

It is assumed in the present study that the screen losses can be neglected. This may be a valid assumption, when proper bonding, etc. is performed, see [1] for confirmation.

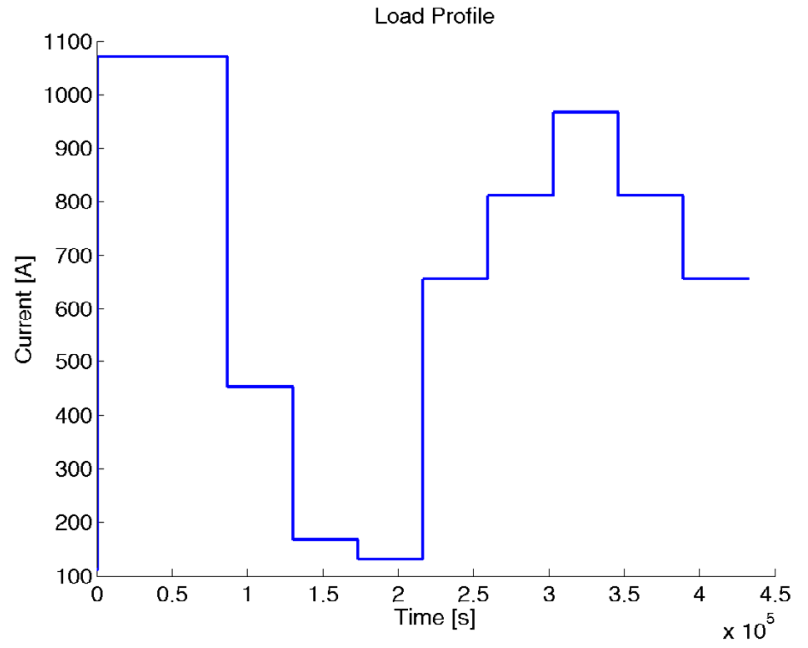

Fig. 4: Load profile of the cable. The cable is seen to be subjected to five days of varying load. Both small and large steps are included in the load profile as well as increases and decreases in the load current are analysed.

On the other hand, for cables operated at voltages at transmission level, dielectric losses cannot be neglected. The present study therefore includes dielectric losses $\left(W_{d}\right)$, and the size is determined by (5) as suggested in [1].

The dielectric losses are estimated to be $11.7 \mathrm{~W} / \mathrm{m}$ for the given cable.

\section{Temperature Profiles}

In this paper a clear distinction is made between temperature distribution and temperature profile. A temperature distribution (dealt with in clause III. D. ) is the geometrical description of the temperature, which means the temperature at different locations in the cable. On the other hand, the temperature profile is concerned with time variations of the temperature at the different sites of the cable.

In Fig. 5 the temperature profile as calculated by the TEE methodology for 1,10 and $100 \pi$-sections is shown. Fig. 5 furthermore shows the same temperature profile calculated by FEM as it can be designed in Comsol.

Fig. 5 shows how sensitive the TEE methodology is to the number of $\pi$-sections which the soil is divided into. It is clear from the figure that a single $\pi$-section is not sufficient for modelling the dynamic thermal behaviour. Modelling with ten $\pi$-sections also seems to deviate from the FEM simulation, although for certain purposes it might be sufficient. One hundred $\pi$-sections are seen to follow the FEM simulation very accurately for the given load profile, and in case of a need for high accuracy, this is the obvious choice among the three shown simulations. Furthermore the method was tested with five hundred $\pi$-sections. However no significant improvement can be seen as compared to one hundred $\pi$ sections. 


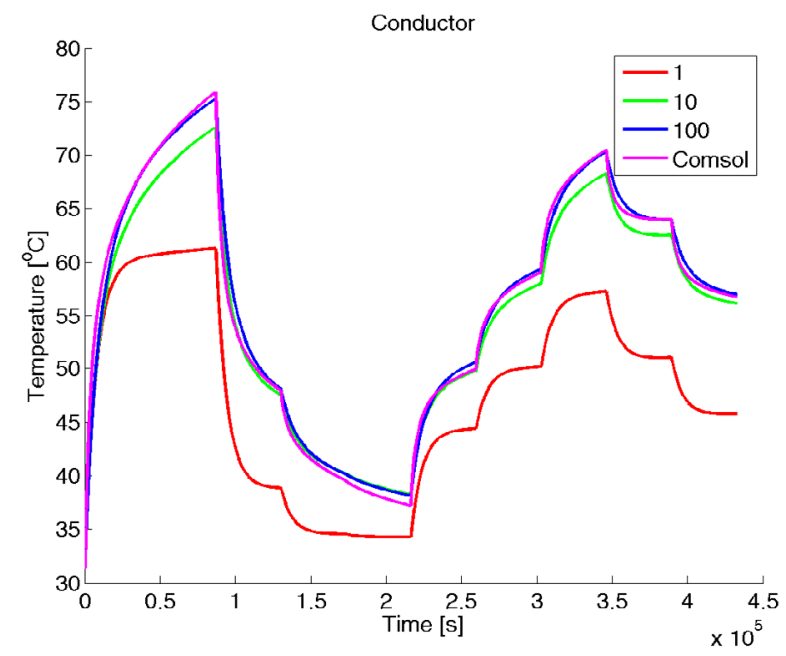

Fig. 5: Temperature profile at the conductor of the $420 \mathrm{kV}$ cable under test. It is seen that the number of $\pi$-sections $(1,10$ and 100) in the thermo electric equivalent has a high impact on the accuracy of the results. The results of the TEEs are here compared with results obtained from the FEM model of Fig. 2.

The drawback of a comparable high number of $\pi$-sections is, as previously discussed, the increased computational time. Fig. 6 shows the computational time as a function of the number of $\pi$-sections. It is seen from the figure that the relation is not linear; however the computational time is only increased approximately two and a half times when the number of $\pi$-sections is doubled.

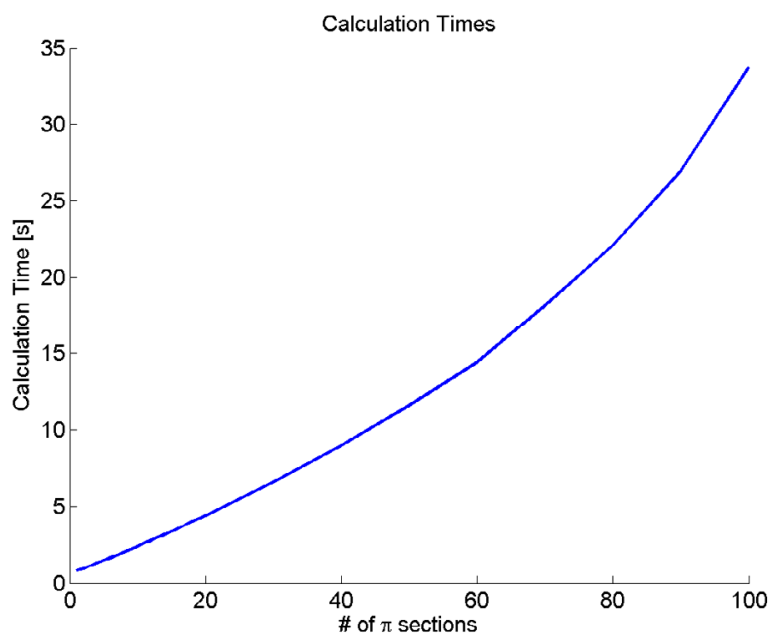

Fig. 6: Computational time for the TEE as a function of the number of $\pi$ sections in the model.

It should be noted that the FEM simulation performed in Comsol Multiphysics took more than 11 minutes of computational time to solve the problem defined in Fig. 2, while, as shown in Fig. 6, the TEE simulations with $100 \pi$ sections only took 35 seconds. Increased speed of the FEM simulations can be obtained by utilising the vertical symmetry around the centre cable, but it is expected that such a symmetrical analysis will only halve the computational time.

Based on the above observations it was decided to model the soil by using one hundred $\pi$-sections. In case of a need for fast calculations, Fig. 5 shows that ten $\pi$-sections can give fairly accurate results.

\section{Temperature Distribution}

The temperature distribution is, as mentioned, a measure of the temperature at different sites in the cable system. Fig. 7 shows the temperature distribution at time $t=0 \mathrm{~s}$ for the cable modelled in Fig. 5.

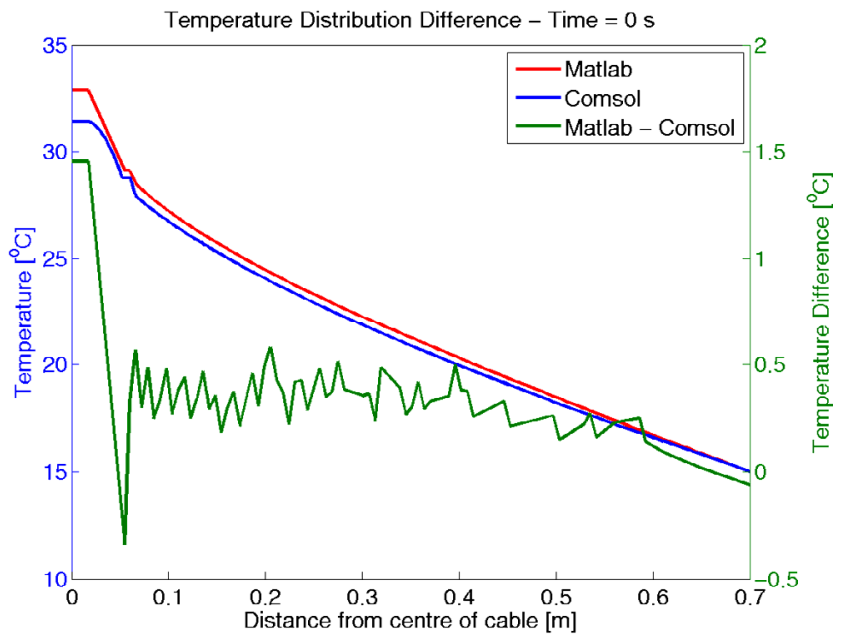

Fig. 7: Temperature distribution from the centre of the conductor, of the centre cable, to ground surface. Some differences are seen in the figure between the TEE model and the FEM model. The shown geometric temperature distribution is a snapshot at $t=0 \mathrm{~s}$ as it is calculated for the load profile of Fig. 4.

It is seen in the figure that the temperature distribution modelled with TEE is very similar to that of the FEM simulation, showing deviations of maximum $1.5^{\circ} \mathrm{C}$.

A similar accuracy in the TEE calculations can be found in Fig. 8. The figure, which shows the temperature distribution at the time $2 \times 10^{5} \mathrm{~s}$ (approximately two days and seven and a half hours), and proves that the TEE methodology enables fast and accurate calculations, deviations are below $1.5^{\circ} \mathrm{C}$, of temperature distributions even under dynamic load conditions.

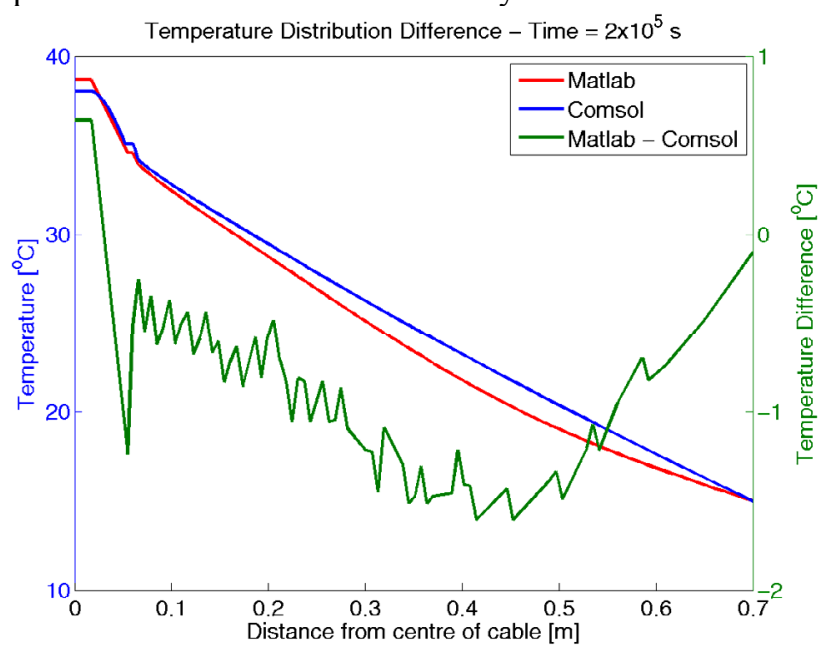

Fig. 8: Temperature distribution at time $t=2 \times 10^{5} \mathrm{~s}$. It is seen that the TEE methodology enables accurate calculations of dynamic temperature distributions.

It has to be emphasised that increased accuracy can be obtained without the need for major changes in the 
methodology. One of the inaccuracies lies in the calculated thermal resistances as calculated by the IEC standard. These inaccuracies are not further dealt with in this paper, as the inaccuracies could just as easily be in the FEM simulations.

Moreover it should be remembered that the thermal capacitance of the soil is approximated with a cylindrical shape which is not quite accurate.

Dealing with both of these issues is left for future research activities.

\section{E. Temperature Estimation and Prediction}

A difference exists between temperature estimation and temperature prediction.

Temperature estimation is concerned with estimating the temperature based on measurements of the current. This quantity can tell about the history of the thermal conditions of the cable, as well as determine whether an existing thermal state of the system is acceptable or not. Any inaccuracies in the temperature estimation thus lie in either the current measurements (not likely with today's SCADA systems) or in the thermal model.

Temperature predictions are concerned with the future, and predictions about the load profile are therefore necessary. The uncertainties in the temperature predictions are therefore at least two-fold, as they include both uncertainties in calculating the temperature and in the predictions of the load profile.

From the calculations point of view, it makes no difference whether the data input are existing measurements or predictions, and the same formulae can therefore be applied.

\section{F. Loadability vs. Time}

An obvious application of the methodology described, is to determine the emergency rating of transmission cables very quickly. This includes schemes which tells the operator of the cable how much he can overload the cable under the given conditions and for how long. Such calculations can be easily performed by using the TEEs.

If it for instance is assumed that the history of the temperature is as given in Fig. 5. At the end of the given temperature profile, a fault happens in the transmission system, causing overloading of the cable. The operator now has to know how much and for how long he can overload the cable. The TEE methodology described in this paper will, within less than one minute, be able to display Fig. 9, informing the operator about the available time for reaction and reconfiguration of the system.

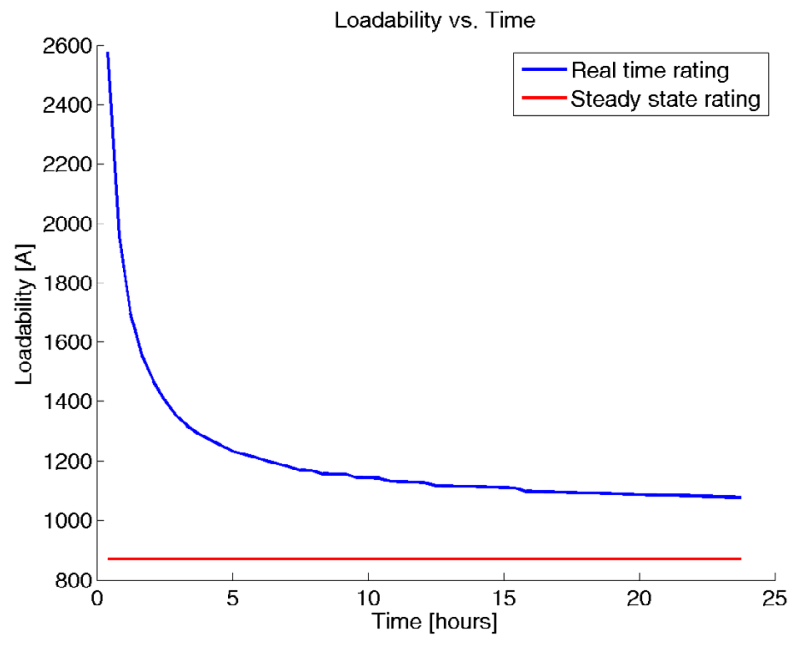

Fig. 9: Loadability of the cable vs. time. During a fault in the transmission system which causes an overloading of the cable, the figure will help the system operator to make proper decisions about how fast he needs to take action in order to prevent damage to the cable.

Faster calculations can of course be made by using fewer $\pi$ sections as a first response to a failure, and by including proper safety margins in the analysis, this might be sufficient. After such a fast, preliminary calculation, the more accurate one hundred $\pi$-section calculations and an even longer timeline, e.g. two weeks, can be given.

The latter is especially important when dealing with cable grids, as repair times usually are not measured in days but in weeks.

\section{CONCLUSION}

The present study has introduced the basic ideas behind the use of fast thermo electric equivalents (TEEs), also denoted the lumped parameters model, within dynamic temperature simulations of transmission cables. The TEE methodology has shown to be capable of making accurate calculations when comparing to a finite element model (FEM), even for highly dynamic load variations.

In order to develop the true dynamic methodology, the authors had to adapt changes to the internationally accepted standard way of calculating the thermal resistance of the cable surroundings. Especially when dividing the cable surroundings into many $\pi$-sections, the methodology proved very accurate, while maintaining a low computational time.

The TEE methodology in the paper is shown to be capable of accurately determining both temperature distributions (geometric dependent) and temperature profiles (time dependent). This enables the operator of the cable system to supervise the current thermal state of the transmission system.

It is moreover shown in this paper that the TEE methodology is capable of helping the operator when failures occur in the system and overloading of the cable is necessary. The TEEs enables a reliable overview of how long and how much the cable can be loaded without risking thermal damage to the cable. 


\section{REFERENCES}

[1] IEC Electric Cables - Calculation of the Current Rating -Thermal Resistance - Calculation of Thermal Resistance. International Electrotecnical Commission Standard 60287-2-1, edition 1.1, November, 2001.

[2] Y.C.Liang and Y.M.Li, "On-line dynamic cable rating for underground cables based on dts and fem," WSEAS Transactions on Circuits and Systems, vol. 7, No. 4, 229-238, April, 2008.

[3] M. Schmale, H.-J. Dräger, and R. Puffer, "Implementation and operation of a cable monitoring system in order to increase the ampacity of a 220kv underground power cable,". 2010 Cigre Session Papers', 21, rue d'Artois, F-75008 Paris, 2010. Cigre. B1-113-2010.

[4] Jerzy Gołębiowski and Marek Zaręba, "The simplified method for transient thermal field analysis in a polymeric DC cable," Electrical Engineering, Springer, vol. 93. No. 4, p. 209-216, May 2011.

[5] IEC Calculation of the cyclic and emergency current rating of cables Part 2: Cyclic rating of cables greater than 18/30 (36) $\mathrm{kV}$ and emergency ratings for cables of all voltages. International Electrotecnical Commission Standard 60853-2, edition 1.0, July, 1989

[6] Ole Christensen, Differentialligninger og uendelige rækker, Technical University of Denmark - Institut for Matematisk Modellering, 2006.

[7] IEC Electric Cables - Calculation of the Current Rating - Thermal Resistance - Calculation of Thermal Resistance. International Electrotecnical Commission Standard 60287-2-1, edition 1.1, Nov., 2001.

[8] G. J. Anders, Rating Of Electric Power Cables, IEEE Press, 1997.

[9] NKT cables, Produktkatalog Elforsyning 2010, [Online], Available: http://www.nktcables.dk/ /media/Denmark/Files/Catalogue/Elforsyning \%20katalog\%202010.ashx, Nov., 2011.

\section{BIOGRAPHIES}

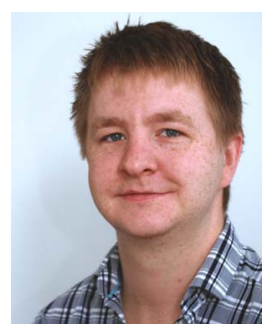

Rasmus Schmidt Olsen received his master of science in electrical engineering in 2010 from the Technical University of Denmark (DTU). His PhD project, which he began shortly thereafter, is concerned with dynamic load optimisation of cable based transmission grids and it is a collaboration between Energinet.dk and DTU.

His main interests are within thermal performance of transmission cables, how this performance can be increased and how asset management can be introduced for a more optimal utilisation of such cables and cable systems.

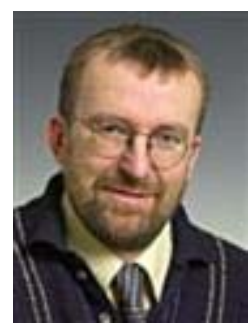

Joachim Holboell is associated professor and group leader at DTU, Department of Electrical Engineering. His main field of research is high voltage components, their properties, condition and broad band performance, including insulation systems performance under AC, DC and transients. Focus is also on wind turbine and future power grid applications.

J. Holboell is Senior Member of IEEE.

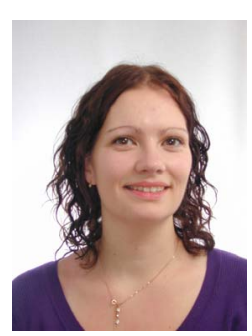

Unnur Stella Guðmundsdottir received her M.Sc. and $\mathrm{PhD}$ degree in 2007 and 2010 respectively, from Aalborg University in Denmark. Her PhD studies focused on modelling of underground cable system at the transmission level. She was a guest researcher at SINTEF in Norway in November 2008 and at Manitoba HVDC Research Centre in Canada during June-October 2009. Currently she holds a position as a cable specialist at Energinet.dk where she is a deputy manager for DANPAC, a research project focusing on undergrounding of almost the entire Danish transmission system. U. S. Gudmundsdottir is a Member of IEEE. 